\title{
Polyglandular Autoimmune Syndrome Type IIIB: Hyperthyroidism and Pernicious Anemia, a Reminder of the Past
}

\author{
Ivan E. Ramirez De Oleo ${ }^{a}$,, Mateo Mejia Saldarriaga ${ }^{a}$, Michael Silverman", \\ Santiago Thibaud ${ }^{\mathrm{b}}$, Luke R. Sponholz ${ }^{\mathrm{b}}$
}

\begin{abstract}
Polyglandular autoimmune syndrome (PAS) refers to the coexistence of at least two different glandular autoimmune diseases. There are several different classes of PAS which involve different organs and present at different age ranges. PAS type IIIB, also known as thyrogastric syndrome, refers to the presence of thyroid autoimmune disease in patients with pernicious anemia. We report a case of a 46-year-old female with Graves disease who presented with pyelonephritis and was found to have severe vitamin B12 deficiency due to pernicious anemia. In patients with autoimmune conditions, it is important to be on the lookout for the development of other forms of autoimmune disease.
\end{abstract}

Keywords: Pernicious anemia; Intrinsic factor; Pancytopenia; Neutropenia; Polyglandular autoimmune syndrome

\section{Introduction}

Pernicious anemia can be part of a series of conditions that share an autoimmune etiology and are grouped under the term polyglandular autoimmune syndrome (PAS). This refers to endocrinopathies characterized by the coexistence of at least two glandular autoimmune-mediated diseases. The association of autoimmune thyroid disease and chronic autoimmune gastritis has been described in the past $[1,2]$. However, the exact mechanism linking thyroid and gastric cell dysfunction is still unknown. Here we describe an interesting case that serves as a

Manuscript submitted September 14, 2017, accepted September 25, 2017

aDepartment of Internal Medicine, Albert Einstein College of Medicine/Jacobi Medical Center, Bronx, NY 10461, USA

bDepartment of Internal Medicine, Albert Einstein College of Medicine/Montefiore Medical Center, Bronx, NY 10461, USA

${ }^{c}$ Albert Einstein College of Medicine, 1300 Morris Park Ave, Bronx, NY 10461, USA

${ }^{\mathrm{d} C}$ Corresponding Author: Ivan E. Ramirez De Oleo, Department of Internal Medicine, Jacobi Medical Center, 1400 Parkway, Bronx, NY, USA.

Email: dr.ierdo@gmail.com

doi: https://doi.org/10.14740/jem451w reminder of this heterogeneous condition and exemplifies the usual presentation of this rare syndrome [3].

\section{Case Report}

A 46-year-old woman with past medical history of hyperthyroidism presented to the emergency room complaining of dysuria, hematuria, and fever for 2 days. Symptomatology started with subjective fevers and chills 4 days prior to admission. Two days before presentation, a mild dysuria developed and progressed to severe discomfort along with new onset hematuria.

Patient was diagnosed with Graves disease 2 years prior to admission after she complained of palpitations, weight loss and heat intolerance to her primary care provider, and was found to have a thyroid-stimulating hormone (TSH) of $<0.01$. She was referred to endocrinology and was started on methimazole. Her follow-up thyroid function tests (TFTs) were within normal limits. On review of systems, she complained of fatigue, generalized weakness, back and neck pain, numbness in the hands and feet, a tingling sensation predominantly in her hands and an occasional electrical sensation over her back with the movement of her head and neck.

On initial evaluation in the emergency room, she was febrile, with temperature of $103.5^{\circ} \mathrm{F}\left(39.7^{\circ} \mathrm{C}\right)$, heart rate of 90 , blood pressure of $119 / 66$, respiratory rate of 17 , and $\mathrm{O}_{2}$ saturation of $100 \%$ on room air. Her physical examination was remarkable for tenderness to palpation of the left flank, left groin and costovertebral angle tenderness predominantly over the left side. Initial workup showed a urinalysis positive for red blood cells (RBCs) of 11 - 20/high-power field (HPF), white blood cells (WBCs) of $>100 / \mathrm{HPF}$ with few bacteria and squamous cells. Complete blood count $(\mathrm{CBC})$ revealed a WBC count of $3,300 / \mu \mathrm{L}$ with an absolute neutrophil count (ANC) of $1,200 / \mu \mathrm{L}$, hemoglobin $(\mathrm{Hgb})$ of $6.7 \mathrm{~g} / \mathrm{dL}$ with a mean corpuscular volume (MCV) of $113.3 \mathrm{fL}$, red cell distribution width (RDW) of $27.4 \%$ and platelets (PLT) of $121,000 / \mu \mathrm{L}$. Liver function test (LFTs) showed total bilirubin of $1.3 \mathrm{mg} / \mathrm{dL}$ with a conjugated bilirubin of $0.2 \mathrm{mg} / \mathrm{dL}$, alanine aminotransferase level of $47 \mathrm{U} / \mathrm{L}$ and aspartate aminotransferase level of 77 $\mathrm{U} / \mathrm{L}$. The remainder of tests including basic metabolic panel, phosphorous, magnesium, prothrombin time, activated partial thromboplastin time, lipase, hepatitis virus B surface antigen and antibody, hepatitis $\mathrm{C}$ antibody, and TSH, were within nor- 


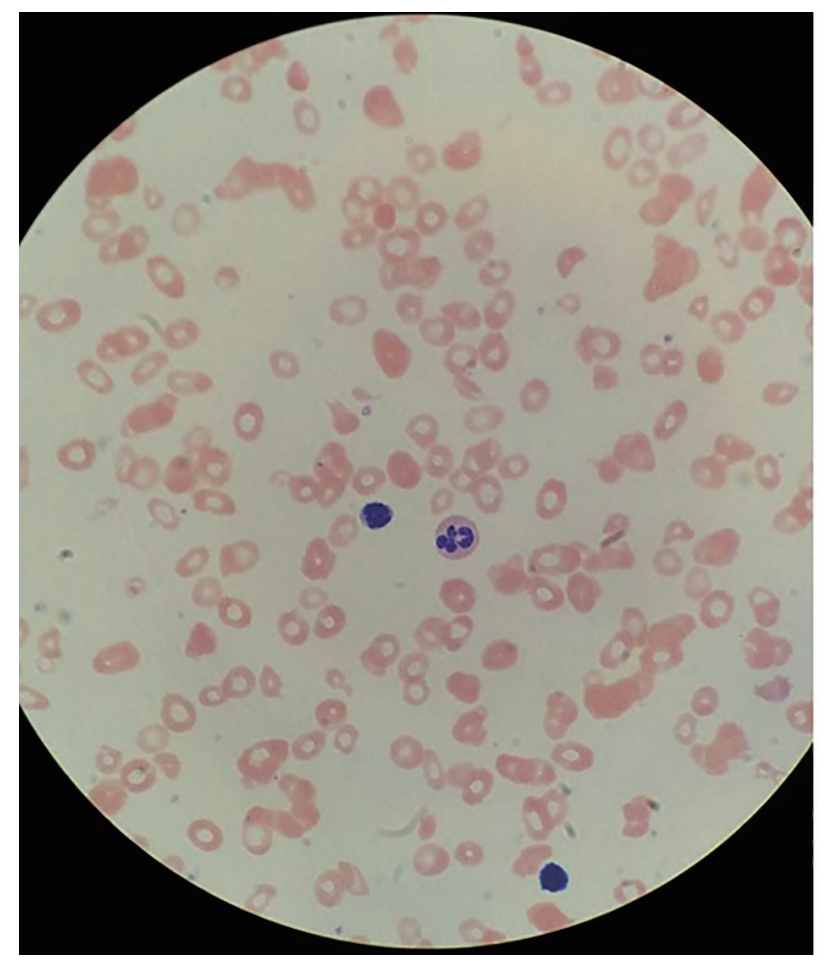

Figure 1. Hypersegmented neutrophil, few platelets, macrocytosis and ovalocytes.

mal limits.

Due to her abdominal discomfort, she was evaluated with a CT scan of the abdomen which showed a distended bladder with fluid, wall thickening and perivesicular inflammatory changes compatible with infectious or inflammatory cystitis. She was admitted to the hospital for pyelonephritis, blood cultures and urine cultures were obtained, and she was started on ceftriaxone $1 \mathrm{~g}$ daily IV.

On day 2 of admission, her Hgb dropped to $5.3 \mathrm{~g} / \mathrm{dL}$, PLT dropped to $70,000 / \mu \mathrm{L}$, and $\mathrm{WBC}$ increased to $4,300 / \mu \mathrm{L}$, but with a decline in the ANC to $400 / \mu \mathrm{L}$. A reticulocyte count was $1.3 \%$ with an absolute count of $18,000 / \mu \mathrm{L}$, and a lactate dehydrogenase (LDH) level was high at 2,475 U/L. Iron studies revealed ferritin of $235 \mathrm{ng} / \mathrm{dL}$, iron level of $42 \mu \mathrm{g} / \mathrm{dL}$, transferrin of $117 \mathrm{mg} / \mathrm{dL}$, saturation of $29 \%$ and total iron binding capacity of $146 \mu \mathrm{g} / \mathrm{dL}$. No evidence of active bleeding was reported. Methimazole was discontinued due to concerns of possible agranulocytosis.

Due to pancytopenia with severe neutropenia and evidence of macrocytosis, a workup for B12 deficiency was sent showing a vitamin B12 level of $<30 \mathrm{pg} / \mathrm{mL}$ (normal: 211 - 946), homocysteine level of $>40 \mu \mathrm{mol} / \mathrm{L}$ (normal: $<15 \mu \mathrm{mol} / \mathrm{L}$ ), methylmalonic acid level of 7,820 nmol/L (normal: 87 - 318), folate level of $12.4 \mathrm{ng} / \mathrm{mL}$ (normal 3.0 - 17.5) and positive intrinsic factor antibody. Peripheral blood smear also revealed macrocytosis, ovalocytes and hypersegmented neutrophils (Fig. 1).

She was diagnosed with pernicious anemia and was started on B12 supplementation with 1,000 $\mu \mathrm{g}$ of vitamin B12 IM daily. The patient was offered RBC transfusion. However, she refused. On day 7 of supplementation, her CBC showed WBC
Table 1. CBC and Reticulocyte Percentage Trend During Hospitalization

\begin{tabular}{lllll}
\hline Variable & Day $\mathbf{1}$ & Day $\mathbf{5}$ & Day 8 & Day $\mathbf{1 1}$ \\
\hline $\mathrm{Hb}(\mathrm{g} / \mathrm{dL})$ & 6.7 & 4.3 & 5.3 & 6.2 \\
$\mathrm{WBC}\left(10^{3} / \mu \mathrm{L}\right)$ & 5 & 5.3 & 5.4 & 4.4 \\
$\mathrm{ANC}\left(10^{3} / \mu \mathrm{L}\right)$ & 0.9 & 0.5 & 0.5 & 1 \\
$\mathrm{Plt}\left(10^{3} / \mu \mathrm{L}\right)$ & 91 & 73 & 95 & 330 \\
Reticulocyte $(\%)$ & - & 1.3 & 8.1 & 15.3 \\
\hline
\end{tabular}

count of $4,400 / \mu \mathrm{L}$ with an ANC of $1,000 / \mu \mathrm{L}, \mathrm{Hgb}$ of $6.2 \mathrm{~g} /$ $\mathrm{dL}$ with an MCV of $116.6 \mathrm{fL}$, an RDW of $26.8 \%$ and PLT of $330,000 / \mu \mathrm{L}$. Reticulocyte percentage increased from $1.3 \%$ to $15.3 \%$ (Table 1).

She completed a course of antibiotics for pyelonephritis without events. Urine and blood cultures were negative after 5 days of incubation. Her hospital course was uneventful. Her dysuria, hematuria and abdominal pain resolved. She was discharged to continue with vitamin B12 supplementation once a week for 7 weeks IM and once a month thereafter. She was started on folic acid and iron supplementation and methimazole was reinitiated for hyperthyroidism. She was discharged in stable clinical condition with hematology follow-up.

\section{Discussion}

Vitamin B12 is an essential element in a series of enzymatic events necessary for the proper development of erythroid, myeloid and megakaryocyte cell lines. Specifically, it is a cofactor of the enzymes methionine synthase and L-methylmalonyl-coenzyme A mutase. It is critical for the development of mature nuclei as well for myelination of the central nervous system [4]. Vitamin B12 deficiency is a major cause of megaloblastic anemia and peripheral neuropathy. The dorsal and lateral columns of the spinal cord are most profoundly affected, leading to sensory loss, paresthesia, and hyporeflexia. Less common associated conditions include thrombosis, infertility, glossitis, and malabsorption [5].

The most common cause of vitamin B12 deficiency worldwide is pernicious anemia, an autoimmune disorder in which there is an immune response against the gastric $\mathrm{H} / \mathrm{K}$-ATPase in the parietal cells in the stomach. This leads to a decrease in the synthesis of intrinsic factor, which is produced by parietal cells in the gastric mucosa. Intrinsic factor binds to ingested vitamin B12 and is necessary for its absorption [6]. The diagnosis of pernicious anemia can be confirmed with laboratory evidence of anti-intrinsic factor antibodies in the serum [7], though this test has low sensitivity but high specificity [8].

Vitamin B12 deficiency, and its most common cause pernicious anemia, can be part of a series of conditions that share an autoimmune phenomenon and are grouped under the term PAS. This refers to endocrinopathies characterized by the coexistence of at least two glandular autoimmune-mediated diseases [2].

Autoimmune thyroid disease, Addison's disease and type 
1 diabetes (T1D) are among the diseases that can be part of the spectrum of PAS [9]. There are three basic subtypes of PAS. Neufeld and Blizzard developed the first classification of PAS in 1980 [1]. The two major subtypes are PAS type I and type II which are characterized by the presence of adrenal cortical involvement. Type I is very rare and presents in infancy or early childhood. It is associated with chronic mucocutaneous candidiasis and harbors a germline mutation in the AIRE gene. Type II (also known as Schmidt's disease) presents in adulthood with the presence of adrenal involvement, T1D, and thyroid disease [1].

PAS type III refers to a subtype that is similar to types II but spares the adrenal cortex. Type III PAS is inherited with a polygenic inheritance pattern and there has been evidence of family clustering of this disorder with several possible permutations of organs involved [2]. The peak incidence is between the ages of 20 and 60 and there can be many years separating the onset of different PAS illnesses. Many different autoimmune diseases are associated with PAS with the most common ones being thyroid disease (70-75\%), T1D (50-60\%), and Addison's disease (40\%) [9]. Type III PAS can be further subdivided into three categories. PAS IIIA refers to autoimmune thyroiditis with T1D. PAS IIIB refers to autoimmune thyroiditis with pernicious anemia. PAS IIIC refers to autoimmune thyroiditis with vitiligo or alopecia.

PAS type IIIB, also known as thyrogastric syndrome, was first described in the early 1960s and refers to the presence of thyroid autoantibodies in patients with pernicious anemia [3]. The follicular thyroid cells and the parietal cells both have endodermal origins, are both polarized, are both characterized by the presence of apical microvilli housing enzymatic activities, and both transport iodine across the cell membrane through $\mathrm{Na}+/ \mathrm{I}-$ symporters. These similarities may contribute to their coexisting autoimmune phenomena [10].

Our patient presented initially with Graves disease and 2 years later developed Pernicious anemia. Her presentation is most consistent with PAS IIIB, which includes autoimmune thyroiditis and pernicious anemia. The patient has no evidence of adrenal autoimmune phenomena and therefore her presentation is not consistent with PAS types I or II. PAS often affects more than two organs and in any patient with PAS, it is important to monitor for the development of new autoimmune diseases.

\section{Conclusion}

The present case describes a middle aged woman with PAS
IIIB who presented with pyelonephritis and pancytopenia with severe anemia and neutropenic fever secondary to pernicious anemia. The association of autoimmune thyroid disease and chronic autoimmune gastritis has been described, though the exact mechanism leading to thyroid and gastric cell dysfunction is still unknown. Though PAS is most commonly associated with Addison's disease, diabetes mellitus, and thyroiditis, it is important to recognize that it can present with involvement of less classical organs.

\section{Conflict of Interest}

No conflict of interest exists from any of the authors.

\section{References}

1. Neufeld M, Maclaren N, Blizzard R. Autoimmune polyglandular syndromes. Pediatr Ann. 1980;9(4):154-162.

2. Eisenbarth GS, Gottlieb PA. Autoimmune polyendocrine syndromes. N Engl J Med. 2004;350(20):2068-2079.

3. Cellini M, Santaguida MG, Virili C, Capriello S, Brusca N, Gargano L, Centanni M. Hashimoto's thyroiditis and autoimmune gastritis. Front Endocrinol (Lausanne). 2017;8:92.

4. Stabler SP. Clinical practice. Vitamin B12 deficiency. N Engl J Med. 2013;368(2):149-160.

5. Remacha AF, Souto JC, Pinana JL, Sarda MP, Queralto JM, Marti-Fabregas J, Garcia-Moll X, et al. Vitamin B12 deficiency, hyperhomocysteinemia and thrombosis: a case and control study. Int J Hematol. 2011;93(4):458464.

6. Stabler SP, Allen RH. Vitamin B12 deficiency as a worldwide problem. Annu Rev Nutr. 2004;24:299-326.

7. Green R. Vitamin B12 deficiency from the perspective of a practicing hematologist. Blood. 2017;129(19):26032611.

8. Carmel R. Reassessment of the relative prevalences of antibodies to gastric parietal cell and to intrinsic factor in patients with pernicious anaemia: influence of patient age and race. Clin Exp Immunol. 1992;89(1):74-77.

9. Kahaly GJ. Polyglandular autoimmune syndromes. Eur J Endocrinol. 2009;161(1):11-20.

10. Doniach D, Ro Itt IM, Taylor KB. Autoimmune phenomena in pernicious anaemia. Serological overlap with thyroiditis, thyrotoxicosis, and systemic lupus erythematosus. Br Med J. 1963;1(5342):1374-1379. 